\title{
Review of: "Genetic Dissection of Drought Resistance of Common Bean at the Seedling Stage by Genome- wide Association Study"
}

\section{만}

Potential competing interests: The author(s) declared that no potential competing interests exist.

Wu et al. used GWAS analysis to identify QTLs related to drought stress at the seedling stage of common bean, and identified the function of a candidate gene PVXIP1;2 in response to drought stress by transforming Arabidopsis and common bean.

The manuscript has reached the standard for publication, but before publication, there are some issues in the manuscript that need to be revised:

1. On page 12 , the author described in the manuscript "No evident difference was observed in the MDA content of WT and transgenic plants under well-watered conditions,". However, according to Figure 5d, there are significant differences between L6 and L8 compared to WT under control conditions.

2. In Figure $6 b$, there is no difference in root length of L8 compared to the wild type under the stress of 300 $\mathrm{mM}$ mannitol. The author should check the results with the description in the manuscript.

3. The list of abbreviations should include all abbreviations in the manuscript.

4. Gene names and Latin in the manuscript should be italicized. 